\title{
VIRAL HEPATITIS
}

\section{Variation in genes encoding for interferon $\lambda-3$ and $\lambda-4$ in the prediction of HCV-1 treatment-induced viral clearance}

\author{
Orazio Palmieri ${ }^{1}$, Antonio M. Ippolito ${ }^{1}$, Maurizio Margaglione ${ }^{2}$, Maria R. Valvano ${ }^{1}$, Domenica Gioffreda ${ }^{1}$, \\ Massimo Fasano ${ }^{3}$, Giovanna D'Andrea ${ }^{2}$, Giuseppe Corritore ${ }^{1}$, Michele Milella ${ }^{4}$, Nicola Andriulli ${ }^{5}$, \\ Filomena Morisco ${ }^{6}$, Lydia Giannitrapani ${ }^{7}$, Anna Latiano ${ }^{1}$, Rosanna Fontana ${ }^{1}$, Pietro Gatti ${ }^{8}$, Paolo Tundo ${ }^{9}$, \\ Michele Barone ${ }^{10}$, Raffaele Cozzolongo ${ }^{11}$, Teresa Santantonio ${ }^{3}$ and Angelo Andriulli ${ }^{1}$ \\ 1 Division of Gastroenterology, Casa Sollievo Sofferenza Hospital, IRCCS, San Giovanni Rotondo, Italy \\ 2 Department of Clinical and Experimental Medicine, University of Foggia, Foggia, Italy \\ 3 Clinic of Infectious Diseases, University of Foggia, Foggia, Italy \\ 4 Clinic of Infectious Diseases, University of Bari, Bari, Italy \\ 5 Department of Chemical Pharmaceutics, University La Sapienza, Rome, Italy \\ 6 Gastroenterology, Department of Clinical Medicine and Surgery, University of Naples 'Federico II', Naples, Italy \\ 7 Dipartimento Biomedico di Medicina Interna e Specialistica (DiBiMIS), Università di Palermo, Palermo, Italy \\ 8 DIMO Medical Oncology, University of Bari, Bari, Italy \\ 9 Division of Infectious Diseases, S. Caterina Novella Hospital, Galatina, Italy \\ 10 Gastroenterology Unit, Department of Emergency and Organ Transplantation, University of Bari, Bari, Italy \\ 11 Division of Gastroenterology, 'S. de Bellis' Hospital, IRCCS, Castellana Grotte, Italy
}

\section{Keywords}

chronic hepatitis - HCV - IL28B/interferon lambda-3 gene - interferon lambda-4 gene peg-interferon/ribavirin

\footnotetext{
Abbreviations

$\mathrm{Cl}$, confidence interval; DAAs, direct-acting antivirals; EOT, undetectable serum HCV RNA at the end of treatment; $\mathrm{HCV}$, hepatitis $\mathrm{C}$ virus; HWE, Hardy-Weinberg equilibrium; IFN $\lambda$-3, interferon Lambda 3; IFN $\lambda$-4, interferon Lambda 4; IL28B, interleukin-28B; LD, linkage disequilibrium; non-SVR, non sustained virological response; OR, odds ratio; PCR, polymerase chain reaction; Peg-IFN, pegylated interferon; RBV, ribavirin; RVR, rapid virological response; SNP, singlenucleotide polymorphism; SVR, sustained virological response.

\section{Correspondence \\ Angelo Andriulli, Division of \\ Gastroenterology, Casa Sollievo Sofferenza \\ Hospital, IRCCS, viale Cappuccini 1, San \\ Giovanni Rotondo, Italy \\ Tel: +390882410263 \\ Fax: +390882 835411 \\ e-mail: a.andriulli@operapadrepio.it}

Received 10 September 2013

Accepted 16 November 2013

DOI:10.1111/liv.12411

Liver Int. 2014: 34: 1369-1377

Evidence has accumulated suggesting that the individual ability to clear HCV infection in the context of therapy with peg-interferon (Peg-IFN) and ribavirin

\begin{abstract}
Background \& Aims: In patients with chronic HCV-1 infection, recent evidences indicate that determination of a dinucleotide polymorphism (ss469415590, $\Delta \mathrm{G} / \mathrm{TT}$ ) of a new gene, designated IFN $\lambda-4$, might be more accurate than the $12979860 \mathrm{CC}$ type of the IL28B locus in predicting sustained virological response (SVR) following peg-interferon and ribavirin. In addition, combined genotyping of different SNPs of the IL28B locus was shown to help dissect patients most prone to SVR among those with rs12979860CT. We examined whether single or combined genotyping of two IL28B SNPs, rs12979860 and rs8099917, and ss469415590 variation might improve the prediction of SVR. Results: In the study cohort of 539 patients, 38\% had SVR. The SNPs 12979860CC, rs8099917TT, and rs469415590TT/TT correlated significantly with SVR (68\%, 50\%, and 67\%). Carriers of either the triplotype rs12979860CC_ss469415590TT/ TT_rs8099917TT or the diplotype rs12979860CC_ss469415590TT/TT had the highest SVR rate (72\%). In carriers of the rs12979860 T allele, neither the rs8099917 nor the ss469415590 improved the response prediction. After pooling this finding with data from previous studies, in rs12979860 T heterozygous individuals the co-presence of the rs8099917TT SNP was associated with improved response prediction. Conclusion: In HCV-1 patients, the rs12979860 polymorphism appeared as the hit SNP better predicting response following peg-interferon and ribavirin treatment. Additional ss469415590 or rs8099917 genotyping had no added benefit for response prediction. In the subset of carriers of the rs12979860 T allele, genotyping of the rs8099917 SNP was unhelpful in the present investigation, but may inform clinical prediction of treatment response when our data were pooled with previous investigations.
\end{abstract}

(RBV) partly reflects differences in the genetic makeup of the human host. Since the landmark discovery that variants on chromosome 19 located within a 
genomic block encompassing the interleukin 28B (IL28B) gene, which encodes for interferon lambda-3 (IFN $\lambda$-3), were associated with either spontaneous or treatment-induced viral clearance (1-6), assessment of the host IL28B genotype is increasingly used among HCV genotype-1 patients to inform clinical prediction of treatment outcome (7). Many single nucleotide polymorphisms (SNPs) were found to play a fundamental role in the response to interferon-based therapy (3-7). More specifically, the rs12979860 and rs8099917 SNPs were the two most examined: they were found in linkage disequilibrium each other, and to be similarly informative as host predictors of sustained viral clearance (SVR). In a recent meta-analysis of results from nine studies regarding the correlation of IL28B genotype with SVR in 3110 Caucasian HCV-1 patients, the favourable IL28B genotype CC was present in $41 \%$ of individuals, and $67 \%$ of them achieved SVR compared to $37 \%$ of those with the unfavourable genotypes (8). In addition, rs8099917 was also indicated as the best tagger of the common haplotype associated with SVR (6).

To better elucidate the mechanisms underpinning the host genetic contribution to the Peg-IFN and RBV treatment, two different strategies have been pursued. In the first approach, combined genotyping of different IL28B SNPs were correlated with treatment outcome: while patients carrying rs12979860CC or rs8099917TT variants may reach high SVR rates (9), in rs12979860 T heterozygous individuals the co-presence of the rs8099917TT SNP has been reported to improve response prediction (10). This finding implies that diplotype analysis could help identify more individuals prone to achieve SVR. However, this claim was not validated in a cohort of patients from Italy (11)

A different approach was taken by ProkuninaOlsson et al. who discovered a dinucleotide polymorphism (ss469415590, $\Delta \mathrm{G} / \mathrm{TT}$ ) between interferon IFN $\lambda-3$ and $-\lambda 2$ in a new gene, designated IFN $\lambda-4$, located $3 \mathrm{~kb}$ upstream of and in the same orientation as IFN $\lambda-3$ (12). This dinucleotide variant was in high linkage disequilibrium with rs12979860 and, compared with the latter SNP, strongly associated with $\mathrm{HCV}$ clearance in individuals of African ancestry, but yielded comparable information in Europeans and Asians (12) and in Hispanic (13). At variance, Bibert et al. were able to detect the strong impact of this genetic variation on SVR prediction even in a cohort of European (Swiss) patients (14). Despite the strong linkage disequilibrium in the locus and the biological role released to new IFN $\lambda$-4 gene, it remains to be independently determined the lead marker across the IFN $\lambda$ region as the key determinant for the differences in HCV-1 clearance following standard therapy. All together, previous findings would indicate that either the combination of different IFN $\lambda-3$ variants and/or the use of IFN $\lambda$-4 genotyping would improve patient management better than that afforded by rs 12979860 SNP evaluation.

To externally validate the lead predictive $\operatorname{SNP}(s)$, triplo- or diplotype, able to better picture the SVR, we investigated the rs12979860, ss469415590 and rs8099917 polymorphisms across the IFNL region in an Italian cohort of 539 naive HCV-1 patients who received PegIFN plus RBV. In an attempt to reconcile discrepancies among previous studies on the contribution of genes encoding for IFNL -3 and -4 proteins to SVR, we run a meta-analysis of available data.

\section{Material and methods}

\section{Patients}

Current analysis refers to $539 \mathrm{HCV}-1$ patients extracted from a larger cohort of 1249 patients who were treated at 16 Italian centers from 2005 to 2010 . The full characteristics of the entire cohort have been presented elsewhere (7). Briefly, all patients included were treatment-naïve Caucasians with a diagnosis of HCV-1 chronic hepatitis or compensated cirrhosis. Patients received either Peg-IFN $\boldsymbol{\alpha}$-2b or $\boldsymbol{\alpha}$-2a plus RBV outside of any clinical trial, and the therapeutic regimen administered was according to recommended guidelines (15). All HCV-1 infected patients enrolled in this investigation agreed to donate a blood sample for genotyping, after having signed an informed consent. At the recall visit, information about the latest HCV RNA testing, liver chemistry, and abdominal ultrasound was recorded. This study was approved by the Ethical Committee of 'Casa Sollievo della Sofferenza' Hospital, San Giovanni Rotondo, Italy, and conducted according to provisions of the Declaration of Helsinki and Good Clinical Practice Guidelines.

\section{Methods}

Genomic DNA was extracted from whole blood samples by a standard non-enzymatic method, using the QIAamp DNA Blood Maxi Kit (Qiagen GmbH, Hilden, Germany). Samples were genotyped for the SNPs ss469415590 and rs8099917 using 5'exonuclease TaqMan genotyping assays on an ABI Prism 7900 RealTime polymerase chain reaction (PCR) system, according to manufacturer's instructions (Applied Biosystems, Foster City, CA, USA). Assays for the rs12979860 genotyping were performed at the Department of Clinical and Experimental Medicine, University of Foggia, Italy, whereas ss469415590 and rs8099917 polymorphisms and data management were under the responsibility of the Research Laboratory of the Division of Gastroenterology, at the IRCCS 'Casa Sollievo della Sofferenza' Hospital, San Giovanni Rotondo, Italy. Briefly, genotyping was performed using made-to-order TaqMan assays for rs8099917, whereas using custom-designed primers for ss469415590, as previously reported (12), 
according to manufacturer's instructions (Applied Biosystems, Foster City, CA, USA). The rs12979860 variant was analysed by direct DNA sequencing with an automated procedure using the 3100 Genetic Analyzer (Foster City, CA, USA) and previously described primers (1).

\section{Viral load, and HCV genotyping}

HCV genotyping was performed using the Inno-LiPA assay (Innogenetics, Zwijndrecht, Belgium). Serum HCV RNA was quantified at baseline by reverse transcription-PCR, using the Cobas Amplicor HCV Monitor Test, v 2.0 (Roche, Basel, Switzerland). Qualitative HCV RNA assessment was made at weeks 4, 24, 48 during treatment, and at week 24 after stopping therapy. We then defined successful treatment response or no response according to standard definitions (15), concentrating on SVR, which is the absence of detectable virus at the end of follow-up evaluation.

\section{Statistical analysis}

All data were analysed on the intention to treat basis, using SPSS Statistical Package (v17 Chicago, IL, USA). Genotypic frequencies for all investigated polymorphisms were tested for consistency with the Hardy-Weinberg equilibrium (HWE) by means of the Haploview 4.2 (Broad Institute, Cambridge, MA, USA). Linkage disequilibrium (LD) between markers, haplotype structures and haplotype associations analyses were also performed. Strong linkage disequilibrium was defined as an $r^{2}>0.8$. The association between genetic and clinical data was calculated by means of univariate and subsequently logistic regression analysis. $P$-values of less than 0.05 were considered as significant.

\section{Meta-analysis}

A meta-analysis was undertaken to establish whether the combination of different variants at the IFN $\lambda$-3 locus or at the IFN $\lambda-4$ locus might extend the prediction power for SVR. A PUBMED computer database search of manuscripts published between September 2009 and July 2013 was performed using the keywords: IL28B AND response AND 'therapy' OR 'therapeutics' AND 'HCV'. After reviewing titles and abstracts of pertinent citations reporting treatment outcome of antiviral therapy in relation to the combined evaluation of different IFN $\lambda-3$ or $\lambda-4$ variants were deemed eligible, and full papers read by two investigators (OP, AL) who independently extracted data. Results from the selected studies were pooled and analysed using the Comprehensive Meta-analyses (version 1.0.25; Biostat, Englewood, NJ, USA). The random-effects method was used to estimate Odds Ratio (OR) with 95\% confidence intervals (95\% CI).

\section{Results}

\section{Patients}

The study cohort included 539 Caucasian patients with chronic HCV hepatitis; main patients' characteristics are shown in Table 1. All patients were infected with genotype 1 , received Peg-IFN $\alpha$-2a $(n=292,54 \%)$ or $\alpha-2 b \quad(n=247,46 \%)$ plus RBV at a mean dosage of $14 \pm 2 \mathrm{mg} / \mathrm{kg}$ of body weight, and had virological response determined 6 months after completion of therapy. Standard therapy applied to these patients led to SVR in 204 (38\%), to rapid virological response (RVR) in 120 (22\%), and undetectable serum HCV RNA at the end of treatment (EOT) in $338(63 \%)$ of them. All responder and non-responder patients received therapy for 48 weeks, except when HCV RNA levels were low (400,000 IU) at baseline, and undetectable at treatment week 4 . In this case, treatment duration was 24 weeks.

\section{IFN $\lambda-3$ and $\lambda-4$ genotype distribution}

The distribution of the three SNPs encompassing the IFN $\lambda$ loci in the total cohort of 539 Caucasian patients was as follows: for the rs 12979860 SNP, prevalence rates of CC, CT, and TT genotypes were $22 \%, 61 \%$, and $17 \%$; for the rs8099917 SNP, the values for TT, TG, and GG genotypes were $44 \%, 49 \%$, and $7 \%$; for the ss 469415590 SNP, the rates were $18 \%, 62 \%$, and $20 \%$ for the TT/TT, $\Delta \mathrm{G} / \mathrm{TT}$, and $\Delta \mathrm{G} / \Delta \mathrm{G}$ dinucleotide genotypes respectively. The allele frequencies of the three SNPs were in

Table 1. Demographic and Baseline Characteristics of 539 HCV-1 patients.

\begin{tabular}{|c|c|c|}
\hline & $N$ & $\%$ \\
\hline \multicolumn{3}{|l|}{ Gender $(N, \%)$} \\
\hline Male & 310 & 58 \\
\hline Female & 229 & 42 \\
\hline Age (mean \pm SD) & $55 \pm 12$ & \\
\hline $\mathrm{BMI}($ mean \pm SD) & $26 \pm 4$ & \\
\hline ALT (U.N.L.) (mean \pm SD) & $2.14 \pm 1.4$ & \\
\hline Platelet count (mean \pm SD) & $190 \pm 66$ & \\
\hline \multicolumn{3}{|l|}{ Liver fibrosis (N, \%) } \\
\hline Stage F0-F2 & 314 & 58 \\
\hline Stage F3-F4 & 225 & 42 \\
\hline \multicolumn{3}{|l|}{ Type 2 diabetes $(N, \%)$} \\
\hline Yes & 52 & 10 \\
\hline No & 487 & 90 \\
\hline \multicolumn{3}{|l|}{ HCV genotypes $(N, \%)$} \\
\hline $1 a$ & 82 & 15 \\
\hline $1 b$ & 456 & 85 \\
\hline \multicolumn{3}{|l|}{ Serum HCV RNA levels ( $N, \%)$} \\
\hline$<400.000 \mathrm{IU} / \mathrm{ml}$ & 159 & 29 \\
\hline$\geq 400.000 \mathrm{IU} / \mathrm{ml}$ & 380 & 71 \\
\hline \multicolumn{3}{|l|}{ Type of Peg-IFN (N, \%) } \\
\hline alpha $2 a$ & 292 & 54 \\
\hline alpha $2 b$ & 247 & 46 \\
\hline
\end{tabular}


accordance with the predicted Hardy-Weinberg's equilibrium $(P>0.05)$ only in patients who attained SVR. The linkage disequilibrium was moderate either between rs12979860 and rs8099917 $\left(r^{2}=0.38\right)$, or between ss469415590 and rs8099917 $\left(r^{2}=0.47\right)$ (Fig. 1). The rs12979860 SNP was in strong linkage disequilibrium with ss469415590 $\left(r^{2}=0.81\right)$. The D prime (D') for the rs12979860, ss469415590, and rs8099917 SNPS was $>0.87$.

Predictive value of candidate SNPs for treatment-induced viral clearance

Two hundred and four patients (38\%) of the study cohort attained SVR. The genotypic distribution of the three SNPs between responders and non-responders is shown in Table 2. For the rs12979860 SNP, SVR rates were $68 \%, 31 \%$, and $24 \%$, respectively for CC, CT, and TT genotypes. For the ss469415590 variation, the values were $67 \%$ for patients with TT/TT, $31 \%$ for $\Delta \mathrm{G} / \mathrm{TT}$, and $29 \%$ for $\Delta \mathrm{G} / \Delta \mathrm{G}$. For the rs8099917 polymorphism, SVR rates were $50 \%$ in patients with TT, $30 \%$ for those carrying the TG variant, and 19\% for GG individuals. At

chr19
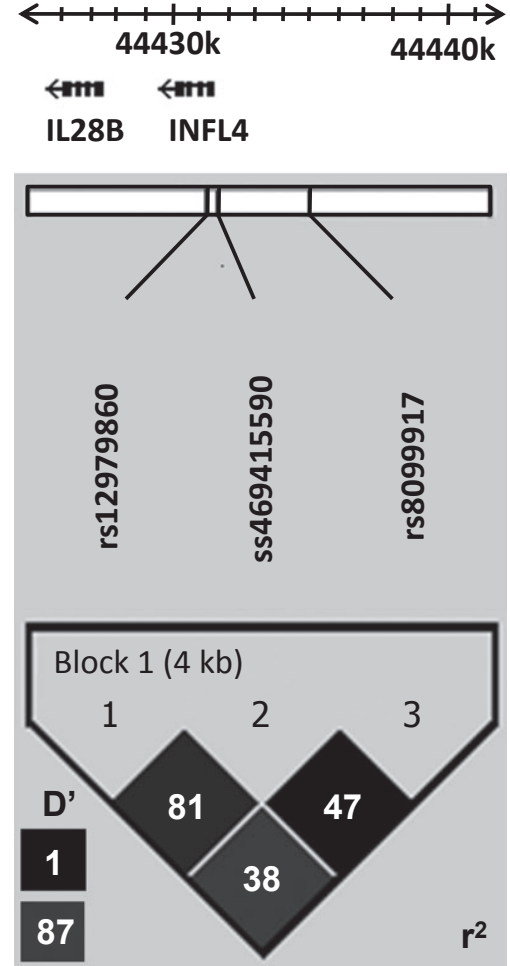

Fig. 1. Linkage disequilibrium (LD) plot for the cohort. $D^{\prime}=$ proportion of possible LD present between the SNPs. D' varies from 0 (complete equilibrium) to 1 (complete disequilibrium) $r^{2}=$ correlation between SNPs. When $r^{2}=1$, two SNPs are in perfect LD and allelic frequencies are identical for both SNPs. The diagram shows the $D^{\prime}$ value in red, whereas the number represent the $r^{2}$. univariate analysis of individual SNPs, genotypes CC for rs12979860, TT/TT for ss469415590, and TT for rs8099917 were all significantly associated with SVR. The prediction power for SVR differed among the three SNPs: the CC homozygous genotype of rs12979860 SNP was more strongly associated (OR $=5.15,95 \%$ CI 3.327.98 for CC vs. CT/TT) than the TT/TT genotype of ss469415590 (OR $=4.73$, 95\% CI 3.02-7.40 for TT/TT vs. $\Delta \mathrm{G} / \mathrm{TT}+\Delta \mathrm{G} / \Delta \mathrm{G})$, or the TT genotype of rs8099917 $(\mathrm{OR}=2.46,95 \%$ CI $1.72-3.51 \mathrm{TT}$ vs. TG/GG).

We next developed a logistic regression model that related clinical and genetic predictors to response rates. In the model including the three favourable genotypes (CC vs. CT/TT, TT vs. GT/ GG, and TT/TT vs. TT/ $\Delta \mathrm{G}+\Delta \mathrm{G} / \Delta \mathrm{G})$, the rs12979860 CC type retained a significant association with SVR (OR $=3.39$; 95\% CI 1.61-7.13), however significance was lost either for the ss469415590 TT/TT (OR $=1.40,95 \%$ CI 0.61-3.21), or for the rs8099917 TT $(\mathrm{OR}=1.34,95 \%$ CI 0.86-2.09). In addition, adjusting for covariates of age, gender, HCV RNA levels, and fibrosis stage, the rs12979860 variant remained the strongest independent predictor of SVR $(\mathrm{OR}=4.72 ; 95 \%$ CI 2.82-7.90).

Combination of IFN $\lambda-3$ and -4 variants and treatmentinduced viral clearance

The impact of the combined evaluation of the two IL28B SNPs, rs12979860 and rs8099917, with the ss469415590 variation on SVR prediction is shown in Table 3. In a triplotype analysis, haplotypes characterized by carriage of the three favourable alleles rs12979860CC_ss469415590TT/TT_rs8099917TT had the strongest association with SVR: $72 \%$ of 99 carriers of the triplotypes attained SVR, as compared with the $30 \%$ SVR rate registered in those with different triplotypes $(\mathrm{OR}=5.85,95 \% \mathrm{CI} 3.61-9.48)$. The strength of the association remained unchanged when the analysis was restricted to diplotypes. In 99 carriers of the rs12979860CC_ss469415590TT diplotype, the SVR rate amounted to $72 \%$, as compared to a value of $32 \%$ in carriers of different diplotypes (OR $=5.85,95 \%$ CI 3.61-9.48). In a similar fashion, the 104 carriers of the rs12979860CC_rs8099917TT diplotype attained a SVR rate of $71 \%$, as compared with the $30 \%$ value registered in those with different diplotypes $(\mathrm{OR}=5.79,95 \% \mathrm{CI}$ $3.61-9.27)$. In addition, SVR rates were $67 \%$ in 110 carriers of the ss469415590TT/TT_rs8099917TT diplotype, and $30 \%$ in those with different diplotypes $(\mathrm{OR}=4.73$, 95\% CI 3.02-7.40).

Predictive value of single or combined SNPs for treatment-induced Rapid Virological Response

One hundred and twenty patients (22\%) of the study cohort attained RVR. The genotypic distribution of the three SNPs is shown in Table 2. For the rs12979860 SNP, RVR rates were $46 \%, 17 \%$, and $9 \%$, respectively 
Table 2. Association between IFN $\lambda$-3 SNPs (rs12979860 and rs8099917) and IFN $\lambda$-4 variation (ss469415590) with Sustained Virological Response (SVR) and Rapid Virological Response (SVR).

\begin{tabular}{|c|c|c|c|c|c|c|c|c|c|c|c|c|c|c|c|c|}
\hline & \multicolumn{2}{|c|}{$\begin{array}{l}\text { Study } \\
\text { cohort }\end{array}$} & \multicolumn{2}{|l|}{ SVR } & \multicolumn{2}{|c|}{ Non-SVR } & \multirow[b]{3}{*}{$P$-value } & \multirow[b]{3}{*}{ OR } & \multirow[b]{3}{*}{ IC 95\% } & \multirow{2}{*}{\multicolumn{2}{|c|}{$\begin{array}{l}\text { RVR } \\
n=120 \\
(22 \%)\end{array}$}} & \multirow{2}{*}{\multicolumn{2}{|c|}{$\begin{array}{l}\text { Non-RVR } \\
n=419 \\
(78 \%)\end{array}$}} & \multirow[b]{3}{*}{$P$-value } & \multirow[b]{3}{*}{ OR } & \multirow[b]{3}{*}{ IC 95\% } \\
\hline & \multicolumn{2}{|c|}{$n=539$} & \multicolumn{2}{|c|}{$\begin{array}{l}n=204 \\
(38 \%)\end{array}$} & \multicolumn{2}{|c|}{$\begin{array}{l}n=335 \\
(62 \%)\end{array}$} & & & & & & & & & & \\
\hline & $N$ & $\%$ & $N$ & $\%$ & $N$ & $\%$ & & & & $N$ & $\%$ & $N$ & $\%$ & & & \\
\hline \multicolumn{17}{|c|}{ rs12979860 } \\
\hline CC & 119 & 22 & 81 & 68 & 38 & 32 & $1.36 \mathrm{e}-14$ & 5.15 & $3.32-7.98$ & 55 & 46 & 64 & 54 & $1.11 \mathrm{e}-12$ & 4.69 & $3.00-7.34$ \\
\hline $\mathrm{CT}$ & 329 & 61 & 101 & 31 & 228 & 69 & & & & 57 & 17 & 272 & 83 & & & \\
\hline $\mathrm{TT}$ & 91 & 17 & 22 & 24 & 69 & 76 & & & & 8 & 9 & 83 & 91 & & & \\
\hline \multicolumn{17}{|c|}{ ss469415590 } \\
\hline$\Delta \mathrm{G} / \Delta \mathrm{G}$ & 98 & 18 & 28 & 29 & 70 & 71 & & & & 11 & 11 & 87 & 89 & & & \\
\hline$\Delta \mathrm{G} / \mathrm{TT}$ & 331 & 61 & 102 & 31 & 229 & 69 & & & & 54 & 16 & 277 & 84 & & & \\
\hline $\mathrm{TT} / \mathrm{TT}$ & 110 & 20 & 74 & 67 & 36 & 33 & $9.87 e-13$ & 4.73 & $3.02-7.40$ & 55 & 50 & 55 & 50 & $4.58 e-15$ & 5.60 & $3.54-8.85$ \\
\hline \multicolumn{17}{|c|}{ rs8099917 } \\
\hline GG & 36 & 7 & 7 & 19 & 29 & 81 & & & & 3 & 8 & 33 & 92 & & & \\
\hline TG & 265 & 49 & 79 & 30 & 186 & 70 & & & & 39 & 15 & 226 & 85 & & & \\
\hline $\mathrm{TT}$ & 238 & 44 & 118 & 50 & 120 & 50 & $5.92 \mathrm{e}-07$ & 2.46 & $1.72-3.51$ & 78 & 33 & 160 & 67 & $1.84 \mathrm{e}-07$ & 3.01 & $1.97-4.59$ \\
\hline
\end{tabular}

$\mathrm{Cl}$, confidence interval; Non-SVR, non sustained virological response; Non-RVR, non rapid virological response; OR, odds ratio; SVR, sustained virological response.

for CC, CT, and TT genotypes. For the ss469415590 variation, the values were $50 \%$ for patients with TT/TT, $16 \%$ for $\Delta \mathrm{G} / \mathrm{TT}$, and $11 \%$ for $\Delta \mathrm{G} / \Delta \mathrm{G}$. For the rs8099917 polymorphism, RVR rates were $33 \%$ in patients with TT, $15 \%$ for those carrying the TG variant, and $8 \%$ for GG individuals. At univariate analysis the studied SNPs were all significantly associated with RVR $(P \leq 1.84 \mathrm{e}-07 ;$ OR $\geq 3.01)$.

By stratifying carriers of the rs12979860CC, ss469415590TT/TT or rs8099917TT, (119, 110 and 208 patients respectively) that achieved RVR, $48(87 \%)$ for rs12979860CC, 47 (85\%), and 78 (85\%) had an SVR. In particular, the achievement of an RVR was related to SVR rates for all the variations, as the SVR rates were $87 \%$ in those with an RVR and $52 \%$ in those without an RVR for rs12979860CC carriers $(P=3.1 \mathrm{e}-05), 87 \%$ vs. $49 \%$ in those ss469415590TT/TT $(P=4.82 \mathrm{e}-05)$, and $85 \%$ vs. $32 \%$ in rs 8099917 TT $(P=4.42 \mathrm{e}-14)$.

The impact of the combined evaluation of the three studied SNPs on RVR prediction is shown in Table 3. In a triplotype analysis, haplotypes rs12979860CC _ss469415590TT/TT_rs8099917TT had the strongest association with RVR, although only 53\% of 99 carriers of the triplotypes attained RVR, as compared with the $15 \%$ RVR rate registered in those with different triplotypes (OR $=6.05,95 \%$ CI 3.78-9.70).

In a diplotypes analysis, in 99 carriers of the rs12979860CC_ss469415590TT diplotype, the RVR rate amounted to $53 \%(\mathrm{OR}=6.05,95 \% \mathrm{CI} 3.78-9.70)$. In a similar fashion, the 104 carriers of the rs12979860CC_rs8099917TT diplotype attained a RVR rate of $52 \%$, as compared with the $12 \%$ value registered in those with different diplotypes $(\mathrm{OR}=6.04,95 \% \mathrm{CI}$ 3.79-9.62). In addition, RVR rates were $50 \%$ in 110 carriers of the ss469415590TT/TT_rs8099917TT diplotype, and $15 \%$ in those with different diplotypes $(\mathrm{OR}=5.60,95 \%$ CI $3.54-8.85)$.

\section{SVR rates in HCV-1 patients with the rs12979860 T allele}

In 329 patients with the heterozygous variant of the rs12979860 SNP, genotyping for the rs8099917 SNP did not improve the prediction of SVR: genotyping for the rs 8099917 SNP yielded 33\% and 30\% SVR rates in TT or TG carriers. In a similar fashion, genotyping for the ss469415590 variant in the same subset of patients yielded $30 \%$ and $30 \%$ SVR rates in TT/TT or $\Delta \mathrm{G} / \mathrm{TT}$ carriers. For the 91 carriers of the homozygous rs12979860 TT non-responder genotype, SVR rates remained unaffected whether the rs8099917 SNP or the ss469415590 variant was evaluated (data not shown).

\section{Meta-analysis of available studies on IFN $\lambda-3$}

Of the 314 published studies on IFN $\lambda-3$ and therapeutic outcome following Peg-IFN and RBV, two studies $(10,11)$ were identified that reported the combined evaluation of the IFN $\lambda$-3 rs12979860 and rs8099917 SNPs and its impact on SVR. Summary data for genotypic distribution of the two IFN $\lambda-3$ SNPs from these studies are shown in supplementary Table 1 and in Table 4: the distribution of either the rs12979860 or rs8099917 SNPs differed among the studies. By pooling SVR data from the studies, genotyping for the latter SNP improved the prediction of treatment-induced viral clearance in the subset of patients with the heterologous $\mathrm{T}$ allele of the rs12979860 SNP: $\mathrm{OR}=1.57$ (95\% CI 1.15-2.17). 


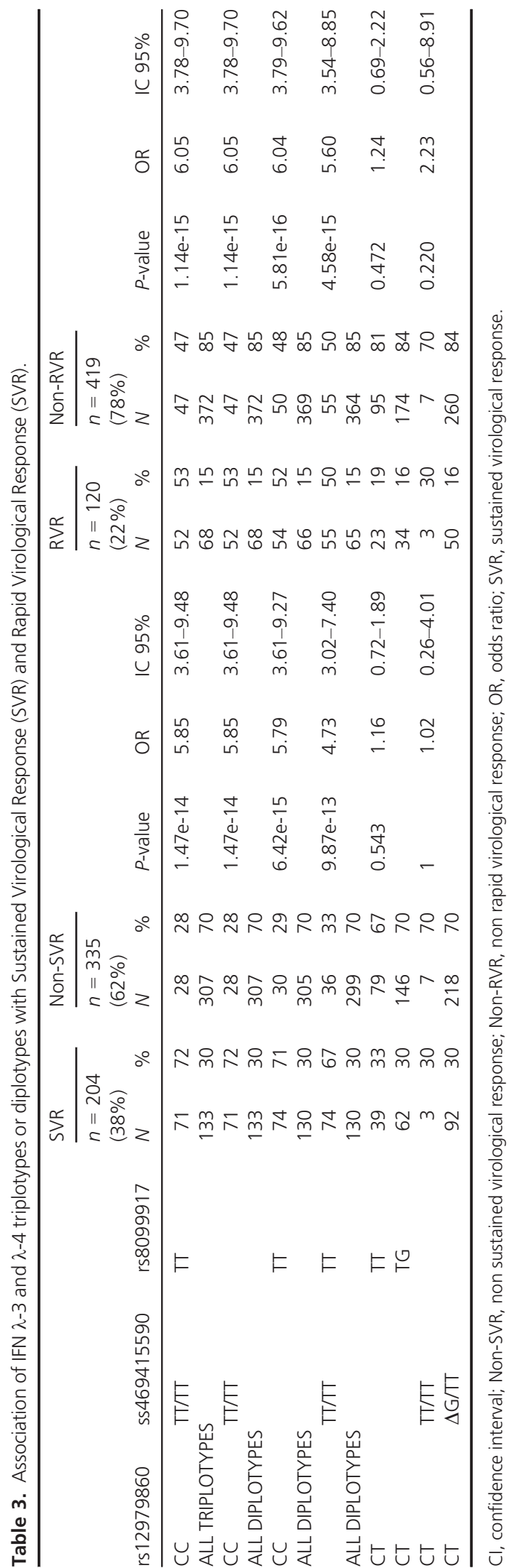

\section{Discussion}

With the approval of the first two direct-acting antivirals (DAAs) protease inhibitors in 2011, several other molecules have been approved for HCV-1 infections. These compounds used in different combinations, till to quadruple therapy, have increased SVR rates to about $90 \%$ (16). In this scenario, IFNL3 and INFL4 genes typing might become appropriate to target patients who could more likely respond to conventional dual therapy with Peg-interferon and ribavirin.

The aim of this study was to establish whether there was an interaction between different IFN $\lambda-3$ and -4 polymorphisms with respect to predicting treatmentinduced clearance in chronic HCV-1 infection. The SNPs evaluated were two variants of the IFN $\lambda$ - 3 locus, rs12979860 and rs8099917, in moderate linkage disequilibrium and likely to be tagging the same haplotype block, and a dinucleotide variation present in the IFN $\lambda-4$, ss469415590TT/TT, which is also in strong linkage disequilibrium with rs12979860 $(12,13)$. These linkage data were confirmed in our cohort of patients.

In the initial analysis of the total cohort of 539 patients, we ascertained the prediction power of each of the three investigated polymorphisms on viral clearance following Peg-IFN plus RBV. The homozygous variants of each genotype were associated with treatment response. At univariate analysis, by genotyping rs12979860CC, ss469415590 TT/TT, and rs8099917TT SVR could be predicted, respectively, in $68 \%$ (81 of 119 ), $67 \%$ (74 of 110), and 50\% (118 of 238) of the study cohort. Therefore, in our Italian cohort the clinical information provided by ss469415590 genotyping appeared comparable to that of the rs12979860 SNP, a finding which is in accordance with the original study by Prokunina-Olsson et al. (12), but at odds with the Bibert et al. (14) who found the TT/-G genotype to predict response to treatment better than the rs12979860. Finally, in our series of patients genotyping the rs8099917 polymorphism was less informative. On the whole, our results would suggest that either the rs12979860 SNP or the ss469415590TT/TT variation might be indifferently genotyped in HCV-1 infected patients before starting antiviral therapy. To establish which of the investigated polymorphisms encompassing the IL28B/IFN $\lambda$-4 locus could more likely be causal, we entered the three polymorphisms into a multiple logistic model: the hit SNP appeared to be the rs12979860CC genotype as it was the only one to retain an independent prediction power $(\mathrm{OR}=3.39$; $\mathrm{CI} 1.61-7.13)$ even when other predictive factors were taken into account.

Our next step was to observe the effect of triplotype/ diplotype in predicting treatment response. In fact, carriers of the triplotype rs12979860CC_ss469415590TT/ TT_rs8099917TT had SVR rate of $72 \%$, a marginally higher value than the $67-68 \%$ rates afforded by the single evaluation of either the rs12979860 SNP or the dinucleotide ss469415590TT/TT variation. However, it 
Table 4. Genotype distribution of single IL28B rs12979860 and rs8099917 SNPs in the cohorts studied in the meta-analysis.

\begin{tabular}{|c|c|c|c|c|c|c|c|c|c|c|c|c|}
\hline & \multicolumn{3}{|l|}{ Present } & \multicolumn{3}{|c|}{ Fischer J. * } & \multicolumn{3}{|c|}{ Fischer J.§ } & \multicolumn{3}{|c|}{ Galmozzi E. } \\
\hline & $\begin{array}{l}\text { All } \\
n=539\end{array}$ & $\begin{array}{l}\text { SVR } \\
n=204\end{array}$ & $\begin{array}{l}\text { Non-SVR } \\
n=335\end{array}$ & $\begin{array}{l}\text { All } \\
n=942\end{array}$ & $\begin{array}{l}\text { SVR } \\
n=495\end{array}$ & $\begin{array}{l}\text { Non-SVR } \\
n=447\end{array}$ & $\begin{array}{l}\text { All } \\
n=377\end{array}$ & $\begin{array}{l}\text { SVR } \\
n=137\end{array}$ & $\begin{array}{l}\text { Non-SVR } \\
n=240\end{array}$ & $\begin{array}{l}\text { All } \\
n=187\end{array}$ & $\begin{array}{l}\text { SVR } \\
n=91\end{array}$ & $\begin{array}{l}\text { Non-SVR } \\
n=96\end{array}$ \\
\hline \multicolumn{13}{|c|}{ rs12979860 } \\
\hline $\mathrm{CC}$ & 22 & 68 & 32 & 34 & 68 & 32 & 26 & 67 & 33 & 30 & 72 & 28 \\
\hline $\mathrm{CT}$ & 61 & 31 & 69 & 52 & 46 & 54 & 55 & 26 & 74 & 57 & 40 & 60 \\
\hline $\mathrm{TT}$ & 17 & 24 & 76 & 14 & 41 & 59 & 19 & 31 & 69 & 12 & 30 & 70 \\
\hline & $\begin{array}{l}\text { reference } \\
17\end{array}$ & & & \multicolumn{9}{|c|}{ rs8099917 } \\
\hline GG & 7 & 19 & 81 & 5 & 35 & 65 & 9 & 31 & 69 & 5 & 30 & 70 \\
\hline TG & 49 & 30 & 70 & 40 & 42 & 58 & 46 & 21 & 79 & 42 & 37 & 63 \\
\hline $\mathrm{TT}$ & 44 & 50 & 50 & 56 & 62 & 38 & 45 & 54 & 46 & 53 & 60 & 40 \\
\hline$P$-value & reference & & & $9 e-5$ & & & 0.31 & & & 0.11 & & \\
\hline
\end{tabular}

Values are given as percentage.

*Discovery cohort.

$\S$ Confirmation cohort.

$\mathrm{Cl}$, confidence interval; Non-SVR, non sustained virological response; OR, odds ratio; SVR, sustained virological response.

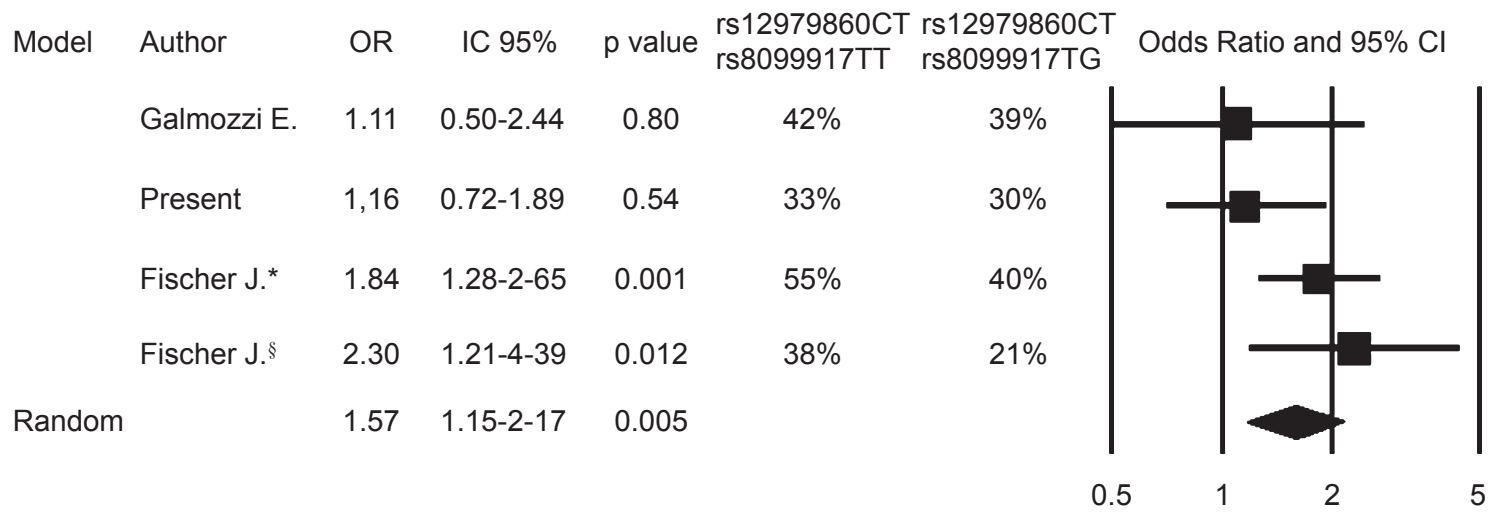

Fig. 2. Meta-analyses of studies that have analysed the rs12979860CT/rs8099917TT diplotype. Fischer et al. *discovery and ${ }^{\S} \mathrm{Confirmation}$ cohorts.

seems most likely that the gain in information provided by the triplotype evaluation was essentially carried over by diplotype analysis. Indeed, equally high rates of SVR $(72-71 \%)$ were registered in carriers of either the rs12979860CC_ss469415590TTTT diplotype and in those with ss469415590TTTT/rs809917TT. Being the modest gain in SVR prediction afforded by either the triplotype or the diplotype analysis, our results would suggest that in homozygous carriers of the rs 12979860 responder $\mathrm{C}$ allele, additional genotyping of the rs8099917 SNP or the ss469415590 variation had no effect on response prediction.

When the week 4 treatment response was taken into account, at univariate analysis rs12979860CC, ss469415590 TT/TT, and rs8099917TT variations were associated with RVR in $46 \%, 50 \%$, and 30\% patients of the study cohort respectively. In addition, the triplotype rs12979860CC_ss469415590TT/TT_rs8099917TT and the diplotype rs12979860CC_ss469415590TT/TT were associated with RVR in 53\% and 53\% of subjects, and comparable predictive value were found also for the other diplotypes combination. It follows that a low utility of genotyping different polymorphisms at the interferon lambda locus, at least for the prediction of an RVR.

A more cogent clinical need is to explore the added clinical value of evaluating different polymorphisms of the IFN $\lambda-3$ and -4 genes in the subset of HCV-1 infected patients carrying the heterozygous rs12979860CT genotype. Although the CC type of this SNP has been consistently shown to best predict SVR (1-5), it has a limited distribution in HCV-1 infected patients $(22 \%$ in the total cohort of the present investigation). The majority of patients carried a non-CC type and exhibited low values of SVR. Consequently, it could be of major clinical interest to verify whether the combined evaluation of more than a single genetic variant could help identify heterozygous rs12979860CT 
patients most prone to achieve a favourable outcome. Of interest, Fischer et al. provided a positive answer to this question by showing that in carriers of the rs12979860 T allele, SVR rates were 55\% in the presence of the rs8099917TT genotype and 40\% in carriers of the rs8099917TG (10). At variance, Galmozzi et al. (11) were unable to replicate the findings in a limited series of patients heterozygous for the rs12979860T allele. In this study, carriers of the rs8099917TT genotype attained SVR rates similar to those seen in rs 8099917 TG carriers ( $42 \%$ vs. $39 \%$ ). The analysis of our large cohort of $539 \mathrm{HCV}-1$ patients produced data in accordance with the Galmozzi' study (11), as heterozygous rs12979860CT patients attained equal rates of SVR whether they were genotyped TT or TG/GG at the rs 8099917 SNP (33\% vs. $30 \% ; P=0.5$ ). We can offer no explanation for the discrepant data from these studies, however would recall the higher SVR rates registered in the Fischer' study in comparison with the rate observed in the present investigations: $53 \%$ vs. $38 \%$.

To solve discrepancies among results from the three studies, we performed a meta-analysis after pooling data from the two previous investigations $(10,11)$ and the present study. As shown in Fig. 2, in more than 1100 heterozygous rs12979860CT patients, the evaluation of the rs8099917 SNP was of clinical utility, as SVR rates were higher $(45 \%)$ in those genotyped TT as compared with a figure of $33 \%$ registered in those genotyped TG $(\mathrm{OR}=1.57,95 \%$ CI 1.15-2.17). However, the metaanalytical results appeared to be driven essentially by the positive data from the Fischer et al.' study (10). We noted a few dissimilarities in patients' features between the present investigation and the German one, in particular in the proportion of those with advanced fibrosis or cirrhosis, which may have impacted on the registered SVR rates and in the overall meaning of our results. We agree with Fischer et al. (17) that more studies on more patients are warranted on this topic.

In conclusion, in the overall population of HCV-1 patients evaluated in the present investigation, the rs12979860 polymorphism at the IL28B locus appeared as the hit SNP better predicting response following PegIFN and RBV treatment: carriers of the homozygous CC genotype attained a $68 \%$ rate of SVR. When evaluated individually, additional ss469415590 or rs8099917 genotyping had no added benefit for response prediction. However, the diplotype rs12979860CC/ ss469415590TTTT was associated with marginally higher rate of SVR (72\%), a gain of seemingly modest clinical utility. Even in the subset of our patients carrying the rs12979860 $\mathrm{T}$ allele, there appears no clinical benefit of evaluating variants at the rs8099917 SNP. However, after pooling this latter results with similar data from previous studies $(10,11)$, we note that in rs12979860 T heterozygous individuals the co-presence of the rs8099917TT SNP was associated with improved response prediction.

\section{Acknowledgements}

Conflict of interest: The authors do not have any disclosures to report.

\section{References}

1. Ge DL, Fellay J, Thompson AJ, et al. Genetic variation in IL28B predicts hepatitis C treatment-induced viral clearance. Nature 2009; 461: 399-401.

2. Tanaka Y, Nishida N, Sugiyama M, et al. Genome-wide association of IL28B with response to pegylated interferon-alpha and ribavirin therapy for chronic hepatitis C. Nat Genet 2009; 41: 1105-9.

3. Suppiah V, Moldovan M, Ahlenstiel G, et al. IL28B is associated with response to chronic hepatitis C interferon-alpha and ribavirin therapy. Nat Genet 2009; 41: $1100-74$.

4. Thomas DL, Thio CL, Martin MP, et al. Genetic variation in IL28B and spontaneous clearance of hepatitis C virus. Nature 2009; 461: 798-801.

5. Rauch A, Kutalik Z, Descombes P, et al. Genetic variation in IL28B is associated with chronic hepatitis C and treatment failure: a genome-wide association study. Gastroenterology 2010; 138: 1338-45.

6. Smith KR, Suppiah V, O'Connor K, et al. International Hepatitis C Genetics Consortium (IHCGC). Identification of improved IL28B SNPs and haplotypes for prediction of drug response in treatment of hepatitis $\mathrm{C}$ using massively parallel sequencing in a cross-sectional European cohort . Genome Med 2011; 3: 57.

7. Andriulli A, Di Marco V, Margaglione M, et al. Identification of naive $\mathrm{HCV}-1$ patients with chronic hepatitis who may benefit from dual therapy with peg-interferon and ribavirin. J Hepatol 2014; 60: 16-21.

8. Rangnekar AS, Fontana RJ. Meta-analysis: IL-28B genotype and sustained viral clearance in HCV genotype 1 patients. Aliment Pharmacol Ther 2012; 36: 104-14.

9. Luo Y, Jin C, Ling Z, et al. Association study of IL28B: rs12979860 and rs8099917 polymorphisms with SVR in patients infected with chronic HCV genotype 1 to PEGINF/RBV therapy using systematic meta-analysis. Gene 2013; 513: 292-6.

10. Fischer J, Böhm S, Scholz M, et al. Combined effects of different interleukin-28B gene variants on the outcome of dual combination therapy in chronic hepatitis $C$ virus type 1 infection. Hepatology 2012; 55: 1700-10.

11. Galmozzi E, De Nicola S, Aghemo A, Colombo M. Is there need for more than one IL28B single nucleotide polymorphism in hepatitis C clinical practice?. Hepatology 2013; 57: 416.

12. Prokunina-Olsson L, Muchmore B, Tang W, et al. A variant upstream of IFNL3 (IL28B) creating a new interferon gene IFNL4 is associated with impaired clearance of hepatitis C virus. Nat Genet 2013; 45: 164-71.

13. Aka P, Kuniholm MH, Pfeiffer RM, et al. Association of the IFNL4- $\Delta \mathrm{G}$ allele with impaired spontaneous clearance of hepatitis C virus. J Infect Dis 2014; 209: 350-4.

14. Bibert S, Roger T, Calandra T, et al. IL28B expression depends on a novel TT/-G polymorphism which improves HCV clearance prediction. J Exp Med 2013; 210: 1109-16.

15. Ghany MG, Nelson DR, Strader DB, et al. An update on treatment of genotype 1 chronic hepatitis $\mathrm{C}$ virus infec- 
tion: 2011 practice guideline by the American Association for the Study of Liver Diseases. Hepatology 2011; 54: 143344.

16. Scheel TK, Rice CM. Understanding the hepatitis $C$ virus life cycle paves the way for highly effective therapies. Nat Med 2013; 19: 837-49.

17. Fischer J, Böhm S, George J, Sarrazin C, Berg T. Impact of cohort size and host factors on combined analysis of interleukin 28B rs12979860 and rs8099917 in hepatitis C virus infection. Hepatology 2013; 57: 416-17.

\section{Supporting information}

Additional Supporting Information may be found in the online version of this article:

Table S1. Baseline Characteristics of HCV Type 1Infected patients according to treatment outcome in present and Fischer et al. 